\title{
Immunoisolation of GABA-Specific Synaptic Vesicles Defines a Functionally Distinct Subset of Synaptic Vesicles
}

\author{
Shigeo Takamori, Dietmar Riedel, and Reinhard Jahn \\ Department of Neurobiology, Max-Planck-Institute for Biophysical Chemistry, D-37077 Göttingen, Germany
}

Synaptic vesicles from mammalian brain are among the best characterized trafficking organelles. However, so far it has not been possible to characterize vesicle subpopulations that are specific for a given neurotransmitter. Taking advantage of the recent molecular characterization of vesicular neurotransmitter transporters, we have used an antibody specific for the vesicular GABA transporter (VGAT) to isolate GABA-specific synaptic vesicles. The isolated vesicles are of exceptional purity as judged by electron microscopy. Immunoblotting revealed that isolated vesicles contain most of the major synaptic vesicle proteins in addition to VGAT and are devoid of vesicular mono- amine and acetylcholine transporters. The vesicles are 10-fold enriched in GABA uptake activity when compared with the starting vesicle fraction. Furthermore, glutamate uptake activity and glutamate-induced but not chloride-induced acidification are selectively lost during immunoisolation. We conclude that the population of GABA-containing synaptic vesicles is separable and distinct from vesicle populations transporting other neurotransmitters.

Key words: GABA uptake; glutamate uptake; neurotransmitter phenotype; vesicular neurotransmitter uptake; synaptic vesicle proteins; vesicular GABA transporter
Synaptic vesicles, the presynaptic storage compartment for neurotransmitters, are presently the best characterized trafficking organelles. More than a dozen membrane proteins that perform specific functions in membrane trafficking are known to be specific to synaptic vesicles (Südhof, 1995). These include the synaptobrevins/vesicle-associated membrane proteins (VAMPs) that mediate membrane fusion, the synaptotagmins that are involved in transmitting the $\mathrm{Ca}^{2+}$ signal to the exocytotic apparatus, synaptophysin, synaptogyrin, and secretory carrier membrane proteins whose functions are not yet known, small GTPases of the rab family that are probably involved in vesicle attachment, and the vacuolar $\mathrm{H}^{+}$-ATPase, which is responsible for intravesicular acidification. Most of these proteins are represented by multiple isoforms that are differentially expressed within the CNS and peripheral nervous system. From numerous immunocytochemical and electron microscopic studies, it can be concluded that at least one member of each protein family is present on every synaptic vesicle within the nervous system (Südhof, 1995).

Despite the similarities in their overall protein composition, synaptic vesicles are distinguished by their neurotransmitter content. The neurotransmitter phenotype is defined by at least three components: the biosynthetic enzyme(s) that generate high concentrations of the transmitter within the presynaptic cytosol, the vesicular carriers specific for the transmitter that are driven by a proton electrochemical potential, and the reuptake systems in the plasma membrane that use the energy provided primarily by the transmembrane sodium gradients (Maycox et al., 1990; Liu and

\footnotetext{
Received Jan. 20, 2000; revised March 27, 2000; accepted April 7, 2000.

We thank Drs. P. De Camilli (New Haven, CT) and K. Buckley (Boston, MA) for providing antibodies. We are grateful to M. Rudnick (New Haven, CT) for her help in raising the VAChT-antibody, W. Jahn and S. Lausmann for their skillful assistance with the light and electron microscopy immunocytochemistry, and Dr. S. Arch for helpful comments and critical reading of this manuscript.

Correspondence should be addressed to Dr. Reinhard Jahn, Department of Neurobiology, Max-Planck-Institute for Biophysical Chemistry, Am Fassberg D-37077 Göttingen, Germany. E-mail: rjahn@gwdg.de.

Copyright (C) 2000 Society for Neuroscience $0270-6474 / 00 / 204904-08 \$ 15.00 / 0$
}

Edwards, 1997; Masson et al., 1999). Transmitter specificity requires an appropriate combination of these components. For instance, the vesicular monoamine transporter transports all monoamines relatively indiscriminately. It then depends on the biosynthetic enzymes whether the neuron is dopaminergic or noradrenergic (Liu and Edwards, 1997). Similarly, the vesicular GABA transporter (VGAT) is also responsible for glycine uptake in glycinergic neurons (Burger et al., 1991; Christensen et al., 1991). Probably, the biosynthetic enzyme glutamic acid decarboxylase (GAD), which is concentrated on the vesicle surface (Reetz et al., 1991), creates high amounts of GABA that are preferentially loaded into the vesicles in GABAergic neurons (Angel and Fleissner, 1983). In contrast, the vesicular glutamate transporter transports glutamate much better than aspartate and thus discriminates between these two transmitter candidates.

In recent years, several of the vesicular neurotransmitter transporters have been characterized at the molecular level (Liu and Edwards, 1997; Masson et al., 1999). In 1992, two genes coding for vesicular monoamine transporters (VMATs) were identified by expression cloning strategies (Erickson et al., 1992; Liu et al., 1992). Furthermore, mutants of Caenorhabditis elegans defective in the vesicular acetylcholine transporters (VAChT) and VGAT (unc-17 and unc-47, respectively) allowed for the cloning of the corresponding genes from nematodes and mammals (Alfonso et al., 1993; McIntire et al., 1997; Sagne et al., 1997). Sequence and structural comparison revealed that the monoamine and acetylcholine transporters are related to each other, whereas VGAT belongs to a different gene family. In agreement with its substrate specificity (see above), VGAT is expressed in both GABAergic and glycinergic neurons (Chaudhry et al., 1998). The vesicular glutamate transporter has not yet been cloned. However, two sodium-dependent transporters specific for proline (Renick et al., 1999) and phosphate (Chaudhry et al., 1998), respectively, are localized preferentially to synaptic vesicles of presumably glutamatergic neurons. The functions of the vesicular pool of these transporters are unknown; they may represent a reserve pool of 
transporters that is incorporated into the presynaptic plasma membranes during high activity.

Presently, it is not known whether vesicles specific for a given neurotransmitter phenotype contain additional specific components. For instance, it has long been known that the shape of synaptic vesicles in inhibitory synapses is different from that of vesicles in excitatory synapses. This difference is evident under certain fixation conditions (Gray, 1959), but its molecular basis is unknown. Furthermore, detailed studies of the mechanisms of vesicular neurotransmitter uptake reveal differences between the individual transporters. For instance, the monoamine and GABA transporters operate as proton exchangers, whereas the glutamate transporter is primarily driven by the membrane potential (Maycox et al., 1990; Liu and Edwards, 1997). In addition, it is unknown how charge and osmotic balance are maintained during transport; it may involve additional elements such as compensatory ion channels. A major problem in identifying such components is caused by the fact that hitherto there has been no method available for isolating neurotransmitter-specific vesicles from mammalian CNS. Thus, the study of transmitter-specific synaptic vesicles was confined to few specialized model systems, such as acetylcholinergic vesicles from electric organs of eels (Whittaker, 1984) and enriched vesicle fractions isolated from peripheral sympathetic nerves.

In the present study, we report the immunoisolation of GABAergic synaptic vesicles from rat brain using an antibody specific for the vesicular GABA transporter. The resulting vesicle fraction is of exceptional purity, $\sim 10$-fold enriched in vesicular GABA transporter but virtually devoid of other vesicular transporters, and functionally active. Our findings document for the first time that GABAergic vesicles are indeed equipped only with the molecular machinery required for GABA uptake and thus are finally responsible for defining the GABAergic phenotype of a neuron.

\section{MATERIALS AND METHODS}

Plasmids and antibodies. The following peptides, all based on the predicted sequence of rat VGAT (McIntire et al., 1997), were synthesized and coupled to keyhole limpet hemocyanin using standard procedures (Schneider et al., 1983): CAEPPVEGDIHYQR [amino acid (aa) residues 75-87, VGAT/1], CDDLDFEHRQGLGD (aa residues 48-61, VGAT/3), and CSLEGLIEAYRTNAED (aa residues 511-525, VGAT/ 4). The sequences corresponding to VGAT/ 1 and VGAT/ 3 are located at the $\mathrm{N}$-terminal cytoplasmic domain, and that corresponding to VGAT/4 is located at the $\mathrm{C}$-terminal cytoplasmic domain. Immunizations and serum collections were performed by commercially available services (Eurogentec, Seraing, Belgium). All antibodies were affinity-purified on the corresponding peptide immobilized via cysteine residue to Thiopropyl Sepharose $6 \mathrm{~B}$ according to the instructions of the manufacturer (Amersham Pharmacia Biotech, Uppsala, Sweden). Antibodies specific for VAChT were generated by the same procedure using a synthetic peptide (CEDDYNYYSRS) as antigen. The rabbit serum was used without further purification.

Antibodies specific for VMAT2 were generated using recombinant protein fragments as antigen and will be described in detail elsewhere (Hoeltje et al., 2000). The following monoclonal antibodies, all available from Synaptic Systems (Göttingen, Germany), were described previously: synaptophysin (Jahn et al., 1985), synaptobrevin (VAMP) 2 (Edelmann et al., 1995), synaptotagmin 1 (Brose et al., 1992), synaptogyrin (Stenius et al., 1995), and syntaxin (Chapman et al., 1995). Furthermore, the following polyclonal antibodies were obtained from Synaptic Systems: synaptobrevin 1, synaptophysin, synapsin, synaptoporin, and the $116 \mathrm{kDa}$ subunit of the vacuolar $\mathrm{H}^{+}$-ATPase. The following antibodies were gifts: GAD (kindly supplied by Dr. P. De Camilli, Yale University, New Haven, CT) and SV2 (kindly supplied by Dr. K. Buckley, Harvard Medical School, Boston, MA).

For transfection experiments, first strand cDNA was generated from
A

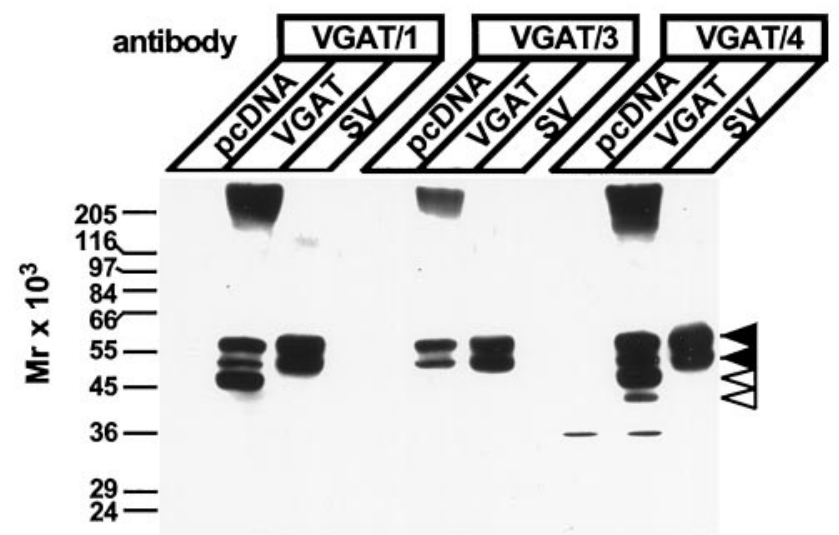

B

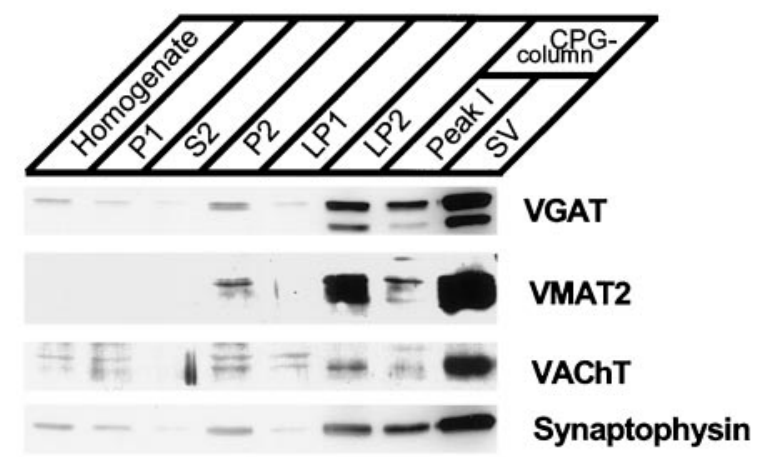

Figure 1. Characterization of antibodies specific for the VGAT. $A$, Antibodies specific for the $\mathrm{N}-(V G A T / 1$ and $V G A T / 3)$ or C-terminal $(V G A T / 4)$ domain recognize identical bands in synaptic vesicles and in cells expressing VGAT. tsA201 cells were transiently transfected with either a rVGAT plasmid $(V G A T)$ or a plasmid without insert ( $p c D N A)$ and analyzed by immunoblotting. For comparison, purified synaptic vesicles $(S V)$ were analyzed in parallel. All three antibodies recognized a doublet band (57 and $50 \mathrm{kDa}$ ) in both synaptic vesicles and transfected cells ( filled arrowheads). Note that some degradation was observed in the heterologous expression system (open arrowheads). B, VGAT copurifies with other vesicular transporters and the vesicle protein synaptophysin during the isolation of synaptic vesicles. Synaptic vesicles were purified using established procedures, with the following fractions being analyzed: Homogenate; $P 1$, crude nuclear pellet; $P 2$, crude synaptosomes $(10,000 \times$ $g$ pellet); $S 2,10,000 \times g$ supernatant; $L P 1,25,000 \times g$ pellet obtained after synaptosomal lysis; $L P 2$, crude synaptic vesicles; Peak 1 and $S V$, large membrane and purified synaptic vesicles as separated by controlled-pore glass $(C P G)$ bead chromatography, respectively (for details, see Huttner et al., 1983; Hell and Jahn, 1994).

rat brain total RNA with SuperScript RT-PCR system and oligo-dT primer (Life Technologies, Gaithersburg, MD). The full-length of rat VGAT was amplified by PCR from the rat brain first strand cDNA using Pfu DNA polymerase and the primers 5'-CGGGATCCATAATGGCCACCCTGCTCCGCAG-3' and 5'-CGTCTAGAGTCCTCTGCGTTGGTTCGGT- ${ }^{\prime}$. The amplified fragment was digested by Bam $\mathrm{HI}$ and $X b a \mathrm{I}$ and was purified by gel electrophoresis. The fragment was subcloned into pcDNA3.1 (Invitrogen, San Diego, CA) at BamHI/XbaI sites, and the construct was then sequenced to confirm the identity with the published sequence (McIntire et al., 1997).

Cell transfection. tsA201 cells, which are HEK293 cells that are transformed to express an SV40 T antigen, were kindly provided by Richard Horn (Thomas Jefferson University, Philadelphia, PA). They were cultured in high glucose DMEM, supplemented with $10 \%$ fetal bovine serum, $100 \mathrm{IU} / \mathrm{ml}$ penicillin, and $100 \mu \mathrm{g} / \mathrm{ml}$ streptomycin. Ten micrograms of either VGAT-pcDNA or pcDNA without insert were used for transfecting cells at $50 \%$ confluence on a $10 \mathrm{~cm}$ dish by means of the calcium-phosphate method. Twenty-four hours after transfection, the 

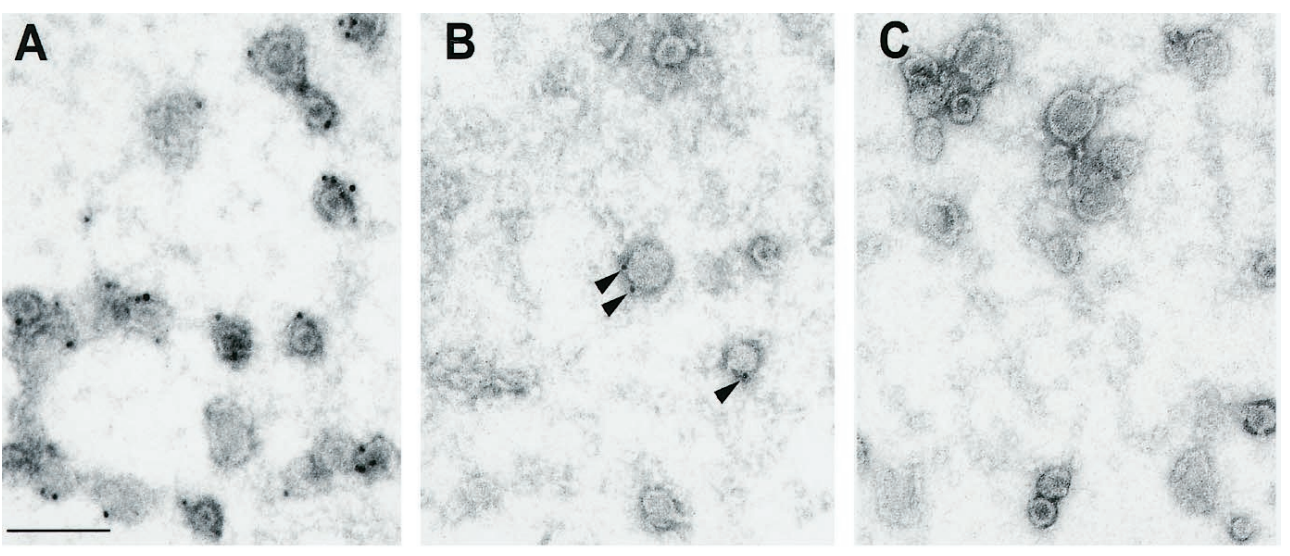

Figure 2. VGAT is present on a subset of synaptic vesicles. Immunogold labeling of synaptic vesicles purified through controlled-pore glass chromatography using anti-synaptophysin antibody (polyclonal) $(A)$, anti-VGAT antibody $(B)$, and preimmune serum $(C)$. $D$ shows an overview of VGAT labeling at a lower magnification. Counting of labeled vesicles from several independent experiments revealed that $\sim 16 \%$ of all small vesicular profiles were labeled with antiVGAT antibody, whereas synaptophysin labeling was observed on virtually all synaptic vesicles. Scale bars: $A, D, 100$ $\mathrm{nm}$. Comparison of the staining patterns for $\operatorname{VGAT}(E, H)$ with those of synaptobrevin $2(F)$ and GAD $(I)$ in sections of rat cerebellum. The staining patterns of VGAT and GAD are identical. In contrast, synaptobrevin 2 antibody stains many more nerve terminals than VGAT; this is particularly obvious in the molecular cell layer $(M O)$. High-magnification overlays of VGAT (red) and GAD (green) $(J)$, and VGAT (red) and synaptobrevin 2 (green) $(G)$ showing Purkinje cell bodies. $G C$, Granular cell layer; $P C$, Purkinje cell layer; $M O$, molecular cell layer. Scale bars: $E, F, H, I, 50 \mu \mathrm{m} ; G, J$, $10 \mu \mathrm{m}$.
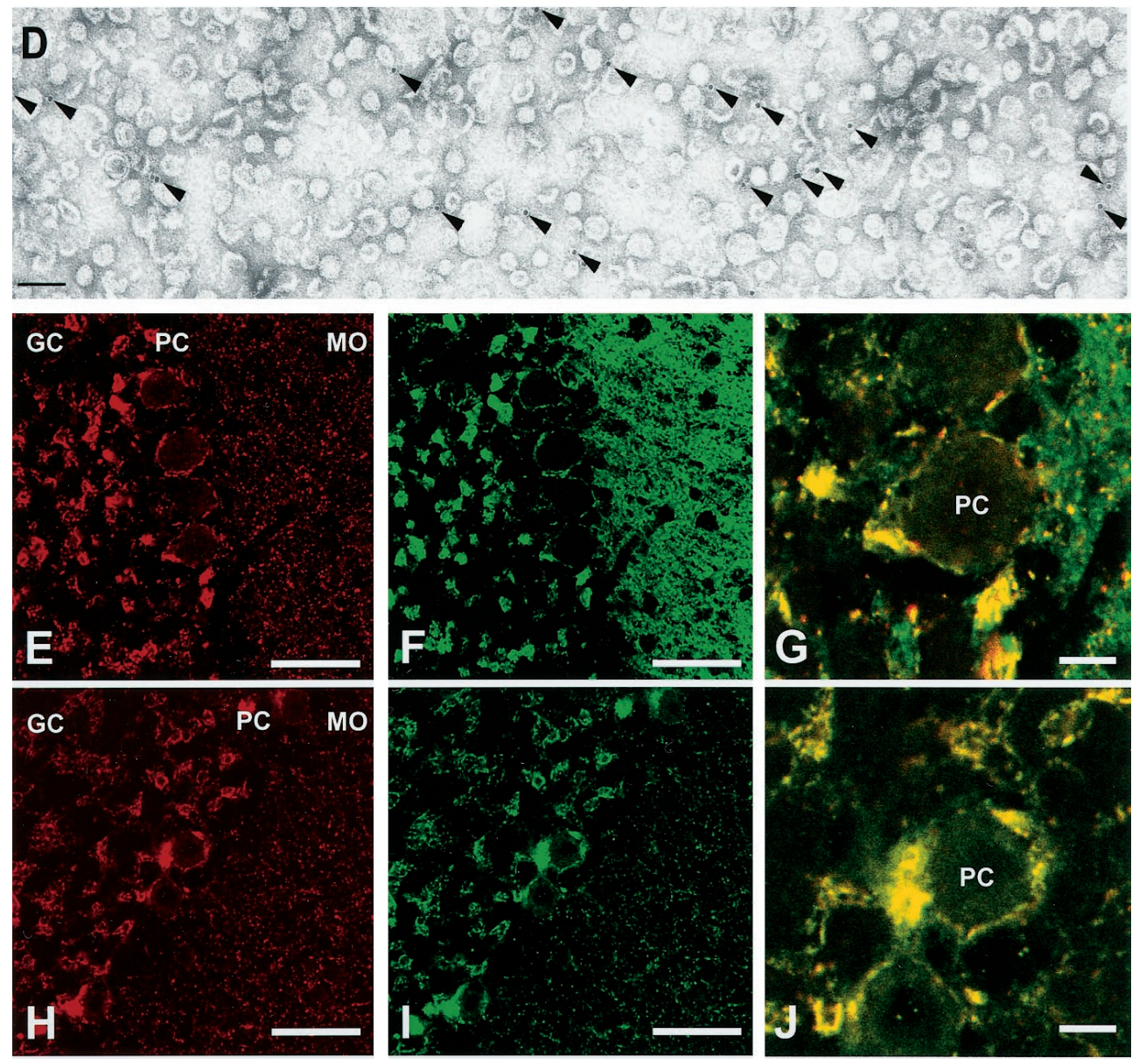

cells were washed twice with ice-cold PBS and then collected. The resulting cell pellet was solubilized in $1 \%$ Triton X-100 containing PBS, and insoluble material was pelleted by $10,000 \times g$ for $15 \mathrm{~min}$. The supernatant was used for immunoblot analysis.

Immunofluorescence. Adult female Sprague Dawley rats were anesthetized, perfused, and post-fixed as described by Mugnaini and Dahl (1983), with modifications. Briefly, a rat was perfused transcardially with ice-cold $0.9 \% \mathrm{NaCl}$, followed by fixative ( $4 \%$ formalin, $0.9 \% \mathrm{NaCl}$, and $0.5 \% \mathrm{ZnCl}_{2}$ ). The cerebellum was dissected, and immersed in the same fixative overnight at $4^{\circ} \mathrm{C}$. After rinsing in $0.1 \mathrm{M}$ Tris- $\mathrm{HCl}, \mathrm{pH} 7.2$, the tissue was transferred to Tris- $\mathrm{HCl}$ containing $20 \%$ sucrose for overnight. Eight micrometer sections were then prepared using a cryostat. The sections were mounted on poly-L-lysine-coated glass slides and dried. Then they were incubated in PBS containing $3 \%$ goat serum and $0.3 \%$ Triton X-100 (GSDB) for $30 \mathrm{~min}$ and then incubated overnight at $4^{\circ} \mathrm{C}$ simultaneously with either affinity-purified anti-VGAT antibody (dilution 1:200)/anti-synaptobrevin monoclonal antibody Cl 69.1 (1:2000), or anti-VGAT antibody/anti-GAD monoclonal antibody (1:50). The sec-
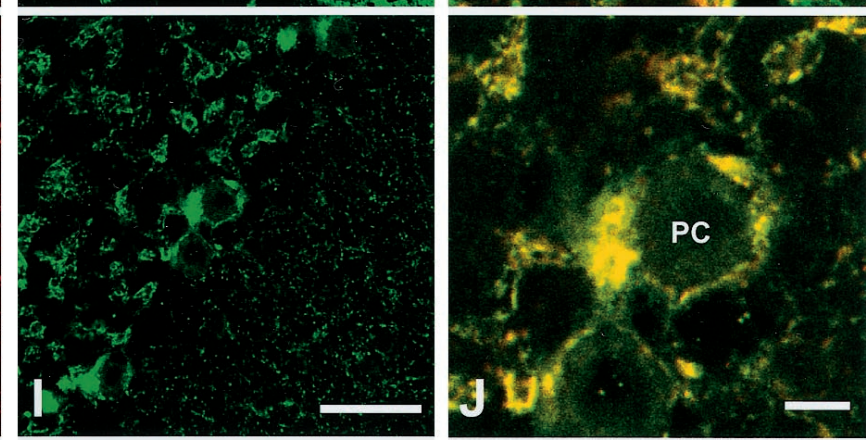

tions were washed with PBS and incubated for $1 \mathrm{hr}$ at room temperature with a mixture of Cy2-conjugated goat anti-mouse antibody and $\mathrm{Cy} 3-$ conjugated goat anti-rabbit antibody $(1: 100$; Jackson ImmunoResearch, West Grove, PA) in GSDB. After being washed with PBS, they were coverslipped with mounting solution (Dako, Glostrup, Denmark) and analyzed by confocal microscopy on an LSM-410-invert (Zeiss, Göttingen, Germany). Images were processed using the Metamorph program package (Visitron, Munich, Germany).

Electron microscopy. The procedure was adapted from De Camilli et al. (1983) and Burger et al. (1989). Beads were pelleted by centrifugation and resuspended in $2 \%$ agarose in $0.1 \mathrm{M}$ cacodylate buffer at $\mathrm{pH}$ 7.4. Thin agarose blocks were cut out, fixed in $2.5 \%$ glutaraldehyde (Electron Microscopy Sciences, Fort Washington, PA) for $1 \mathrm{hr}$ at room temperature, post-fixed with $1 \% \mathrm{OsO}_{4}$ in cacodylate buffer plus $1.5 \% \mathrm{~K}_{3} \mathrm{Fe}_{3}(\mathrm{CN})_{6}$ on ice, followed by a wash with $\mathrm{H}_{2} \mathrm{O}$, and treated with $1 \%$ uranyl acetate. The samples were then dehydrated in ethanol and embedded in EM bed 812. After polymerization at $60^{\circ} \mathrm{C}$ for $48 \mathrm{hr}$, ultrathin sections were cut and stained with uranyl acetate and lead citrate. 

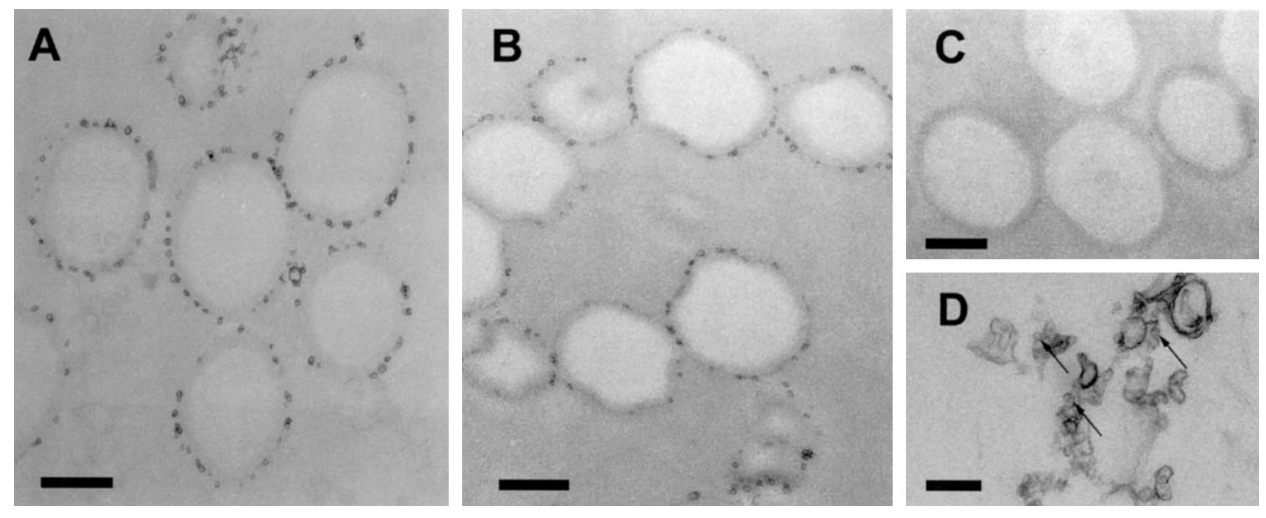

Figure 3. VGAT immunobeads bind a highly homogenous organelle population with the size and shape of synaptic vesicles. $A$, VGAT beads; $B$, synaptophysin beads; $C$, control beads. $D$ shows a representative field of the enriched vesicle fraction (LP2; arrows indicate small profiles of the size of synaptic vesicles) used as starting material for immunoisolation. See Materials and Methods for details. Scale bars, $500 \mathrm{~nm}$.
For immunogold labeling, purified synaptic vesicles were adsorbed to glow discharged grids and fixed with a mixture of $4 \%$ paraformaldehyde and $0.1 \%$ glutaraldehyde in $0.1 \%$ potassium-sodium phosphate buffer, $\mathrm{pH}$ 7.4. Thereafter, labeling with diluted respective antibodies and $6 \mathrm{~nm}$ of protein A gold conjugates diluted at 1:1000 in 1\% BSA in phosphate buffer were performed. The samples were post-fixed for 10 min with $2 \%$ glutaraldehyde in phosphate buffer, washed with $\mathrm{H}_{2} \mathrm{O}$, rinsed with three drops of $1 \%$ uranyl acetate, and immediately dried with filter paper.

Immunoisolation. Affinity-purified VGAT-antibodies (VGAT/1) were conjugated to Eupergit C1Z methacrylate microbeads ( $1 \mu \mathrm{m}$ mean diameter; Röhm Pharmaceuticals, Darmstadt, Germany) as described previously (Burger et al., 1989). Synaptophysin beads and control beads (glycine-inactivated) were prepared as described previously (Burger et al., 1989). LP2 fraction (starting material) was incubated with beads for $2 \mathrm{hr}$ at $4^{\circ} \mathrm{C}$ with constant rotation. After incubation, the beads were sedimented by centrifugation at 10,000 rpm for $1 \mathrm{~min}$ and washed three times with PBS. Unbound membranes in the supernatant solution and the same amount of starting material were pelleted by $80,000 \mathrm{rpm}$ for 20 min in TLA120.2 rotor. All membrane or bead pellets were resuspended in SDS-PAGE sample buffer for gel electrophoresis and immunoblotting, in PBS for electron microscopy, and in sucrose-based buffer (see below) for amino acid uptake assays. Synaptic vesicles were also purified according to a conventional procedure described previously (Hell and Jahn, 1994).

Vesicular GABA and glutamate uptake assay. Uptake assays for glutamate and GABA was performed as described previously (Hell et al., 1988). The fractions obtained by VGAT bead immunoisolation were resuspended in $1 \mathrm{ml}$ of assay buffer $(0.32 \mathrm{M}$ sucrose, $4 \mathrm{~mm} \mathrm{KCl}, 4 \mathrm{~mm}$ $\mathrm{MgSO}_{4}$, and $10 \mathrm{~mm}$ HEPES-KOH, pH7.4). Aliquots (100 $\left.\mu \mathrm{l}\right)$ from each fraction were used for one assay sample. In parallel, the amount of synaptophysin in each fraction was quantified as described previously (Jahn et al., 1984). Uptake activities were normalized to synaptophysin as a measure for the amount of synaptic vesicles.

Acidification assay. Either starting material (LP2) or immunoisolates (synaptophysin beads, VGAT beads, and control beads) were resuspended in acidification assay buffer containing $320 \mathrm{~mm}$ sucrose, $2 \mathrm{~mm}$ $\mathrm{MgSO}_{4}, 2 \mathrm{mM} \mathrm{MgCl}_{2}$, and $10 \mathrm{~mm} 3$-[ $N$-morpholino]propanesulfonic acid, adjusted to $\mathrm{pH} 7.4$ with $\mathrm{KOH}$. The acidification assay for each fraction was performed as described by Hell et al. (1990).

Immunoblot analysis. SDS-PAGE was performed according to Laemmli (1970), and Western blotting was performed according to Towbin et al. (1979). For detection, the appropriate secondary antibody or protein A (both conjugated to horseradish peroxidase; Sigma, St. Louis, MO) was used at dilutions of 1:10,000 and 1:5000, respectively. After washing steps, the horseradish peroxidase was detected by enhanced chemiluminescence using a commercially available kit (Pierce, Rockford, IL). For synaptophysin, SV2, and synaptobrevin 2, a secondary antibody conjugated to alkaline-phosphatase was used (1:3000; Sigma) and visualized by a combination of nitro blue tetrazolium $(0.33 \mathrm{mg} / \mathrm{ml})$ and 5'-bromo-4-chloro-3-indolyl phosphate $(0.17 \mathrm{mg} / \mathrm{ml})$.

\section{RESULTS}

\section{VGAT-specific antibodies recognize a subset of synaptic vesicles}

To characterize GABAergic synaptic vesicles, we generated a panel of serum antibodies specific for VGAT. Rabbits were im- munized with peptides corresponding to regions in the $\mathrm{C}$ - and $\mathrm{N}$-terminal cytoplasmic tails of the transporter, respectively. After affinity purification, three of the sera recognized a major double-band in purified synaptic vesicles with a molecular weight expected for VGAT (McIntire et al., 1997). Bands with an identical mobility were recognized in tsA201 cells transfected with VGAT cDNA, whereas no signal was detectable in mocktransfected cells. Unless indicated otherwise, all subsequent experiments were performed with VGAT/1 antibody.

We compared the enrichment of VGAT with that of other vesicular transporters during the purification of synaptic vesicles from rat brain. Synaptic vesicles were isolated from synaptosomes (P2 fraction) by differential and sucrose-gradient centrifugations, followed by chromatography on controlled-pore glass beads using established procedures (for details, see Huttner et al., 1983; Hell and Jahn, 1994). As shown in Figure 1B, VGAT copurified with VMAT2, VAChT, and synaptophysin, an ubiquitous synaptic vesicle membrane protein of unknown function.

Previous studies have shown that the major synaptic vesicle proteins are present on virtually all vesicles in a purified synaptic vesicle fraction (for review, see Südhof, 1995). Furthermore, it is known that purified synaptic vesicles contain uptake activities for all classical (i.e., nonpeptide) neurotransmitters, suggesting that they are composed of a mixture of vesicles that are distinguished by their respective neurotransmitter transporters. To assess the proportion of synaptic vesicles containing VGAT, we performed immunogold labeling of our purified synaptic vesicle fraction. As shown in Figure $2 A-D$, VGAT immunogold labeling was found only on a subset of synaptic vesicles, whereas virtually all vesicles were prominently decorated with synaptophysin antibodies. We determined the percentage of labeled vesicles in several independent experiments and found that $\sim 16 \%$ of all vesicles in the preparation were positive for VGAT. This number, which is approximately within the expected range for the percentage of GABAergic nerve terminals, did not change when the antibody concentration was varied.

To confirm that the antigen recognized by our VGAT antibody is indeed specific for GABAergic nerve terminals, we compared its distribution with that of the GABA-synthesizing enzyme GAD by light microscope immunocytochemistry. In sections of the rat cerebellum, VGAT staining was prominent around the cell body of the Purkinje cells, particularly in the basket cell synapse at the axon hillock, which is known to be GABAergic (Oertel et al., 1981). Furthermore, staining was also observed in the granular cell layer, whereas in the molecular cell layer, only sparse puncta were observed. The staining for GAD was virtually iden- 


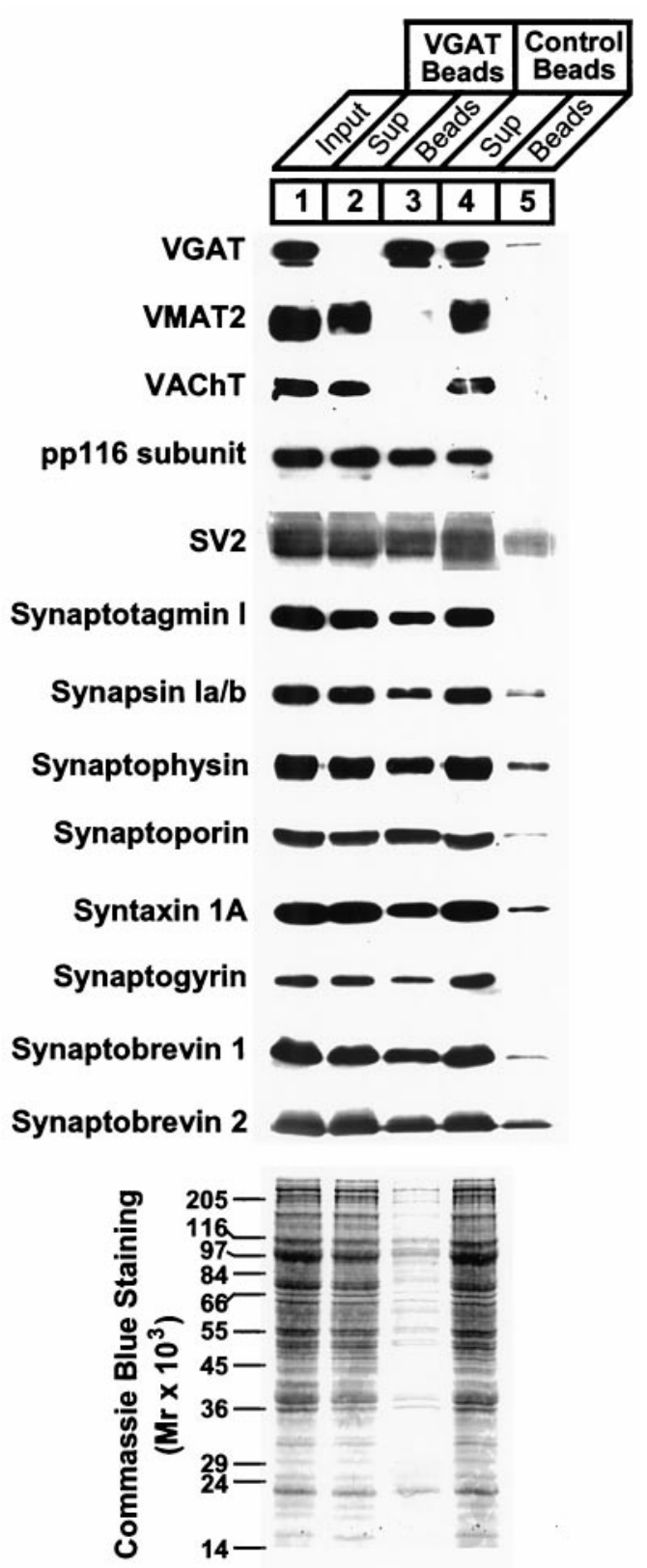

Figure 4. Vesicles isolated on beads containing VGAT antibodies (VGAT Beads) are devoid of VAChT and VMAT2 but contain other synaptic vesicle proteins. The resuspended vesicle fraction (LP2) used as starting material (Input) was compared with the bead-bound material (Beads) and the material remaining in the supernatant after bead incubation (Sup). To allow for direct comparison, equal relative amounts of each fraction were separated in parallel.

tical. This confirms that VGAT and GAD are coexpressed in a subset of neurons (Fig. $2 H-J$ ). In contrast, synaptobrevin, a ubiquitous synaptic vesicle membrane protein, is widespread, particularly in the molecular cell layer, which contains predominantly glutamatergic nerve terminals (Fig. $2 E-G$ ). These findings agree with previous observations (Chaudhry et al., 1998). Under the conditions we used, it is possible to infer that our antibody specifically binds to the cytoplasmic tail of VGAT on the surface of synaptic vesicles.
A
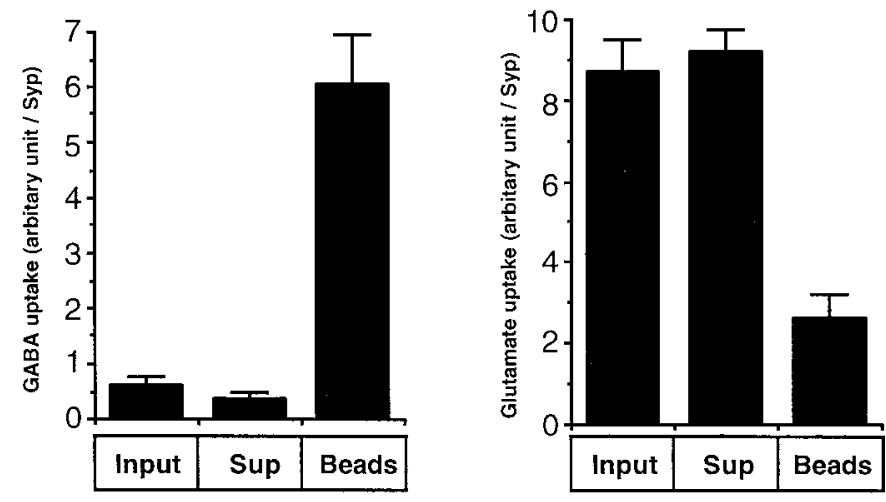

Figure 5. VGAT immunoisolates are enriched for GABA uptake activity and impoverished for glutamate uptake activity. FCCP-sensitive GABA $(A)$ and glutamate $(B)$ uptake activities in LP2 (Input), unbound material (Sup), and bound material on VGAT beads (Beads) were measured using radiolabeled substrates and a standard filtration assay as described in Materials and Methods. All values were normalized to the relative amount of vesicle marker synaptophysin. The figures shows mean \pm SD values of at least two independent experiments (in each experiment, $n=4$ ).

\section{Immunoisolation of GABAergic vesicles defines a functionally distinct subpopulation of synaptic vesicles}

To isolate VGAT-specific synaptic vesicles from rat brain, affinity-purified VGAT/1 antibody was coupled to methacrylate beads (referred as VGAT beads). Methacrylate beads are nonporous and exhibit virtually no nonspecific binding of proteins or membranes (Burger et al., 1989). We showed previously that synaptic vesicles isolated with this procedure using a synaptophysin antibody contain high concentrations of GABA and glycine (Burger et al., 1991). As starting material, we used a resuspended LP2 fraction in PBS (Fig. $1 B$ ) that is approximately eightfold enriched in synaptic vesicles but still contains some contaminating large membranes (Fig. 3D) (Huttner et al., 1983; Jahn et al., 1985). VGAT beads were added to the suspension and, after an incubation for $2 \mathrm{hr}$, were isolated by centrifugation at 10,000 rpm for $1 \mathrm{~min}$. For comparison, incubations were performed in parallel with inactivated beads containing no antibody (control beads) and beads containing synaptophysin antibodies. As revealed by electron microscopy of ultrathin sections of the isolated bead fractions, VGAT beads were uniformly covered with a homogeneous population of vesicular structures with the expected size $(40-70 \mathrm{~nm})$ of synaptic vesicles (Fig. 3A). Very similar results were obtained with synaptophysin beads (Fig. 3B), whereas control beads were devoid of bound membranous vesicular structures (Fig. 3C).

Next, we analyzed the protein composition of the bead-bound membranes by immunoblotting using a panel of antibodies specific for synaptic vesicle proteins. For comparison, the same relative amount of starting material, bead-bound, and unbound material were analyzed by SDS-PAGE and immunoblotting. The results are shown in Figure 4. First, VGAT was almost quantitatively bound to the VGAT beads. Commassie blue staining showed that the VGAT bead fraction contained only a small percentage of starting material with most protein remaining in the supernatant solution. Second, the immunoisolates were completely devoid of VMAT2 and VAChT, demonstrating that no overlap exists between GABAergic and monoaminergic or cho- 

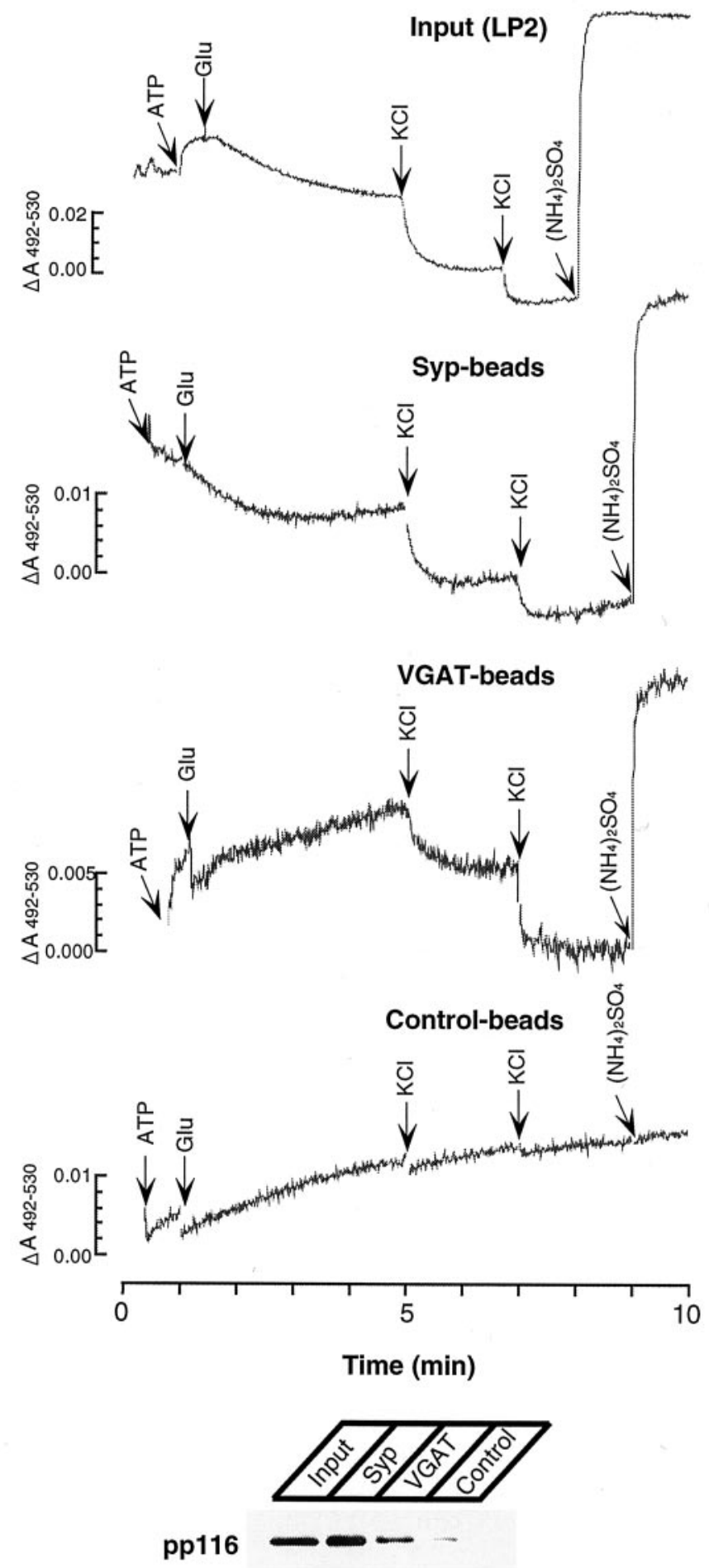

Figure 6. Comparison of ATP-induced acidification using glutamate and chloride as counterion in VGAT and synaptophysin immunoisolates. Acidification was monitored by double-wavelength spectroscopy using acridine orange as indicator dye. The reaction was started by adding ATP. At the end of the reaction, $20 \mathrm{~mm}\left(\mathrm{NH}_{4}\right)_{2} \mathrm{SO}_{4}$ was added to equalize the intravesicular $\mathrm{pH}$ with that of the medium (Hell et al., 1990). The bottom shows an immunoblot for the $116 \mathrm{kDa}$ subunit of the vacuolar proton ATPase for each fraction. Note that, in this experiment, the recovery of V-ATPase was lower in the VGAT bead fraction than in the synaptophysin bead fraction, requiring higher amplification to obtain comparable signals. linergic vesicles. Third, VGAT immunoisolates contained all the major synaptic vesicle proteins tested (Fig. 4). As evident from the figure and further confirmed by quantitative immunoblotting (data not shown), the relative proportions of these proteins were similar to the starting material (Fig. 4) and to synaptophysin immunoisolates (data not shown). Although it has been inferred previously from immunocytochemical studies that GABAergic nerve terminals contain most of these proteins (Südhof, 1995), it was unclear whether these proteins were present in similar proportions as in other synapses. In addition to general trafficking proteins, such as synaptobrevins, synaptotagmins, and syntaxin 1 , these include the vacuolar proton pump (here shown as the 116 kDa subunit), which generates an electrochemical proton gradient, thus providing the driving force for neurotransmitter uptake into synaptic vesicles.

The data presented so far suggest that immunoisolation of VGAT-containing vesicles resulted in a pure GABAergic vesicle fraction with only negligible contamination by vesicles specific for other neurotransmitters. To confirm that the immunoisolates represent a functionally separate subpopulation of synaptic vesicles, we assayed for proton gradient-dependent GABA uptake using a standard transport assay (Hell et al., 1990). Preliminary experiments (data not shown) revealed that the antibody did not inhibit uptake activity, a prerequisite for activity experiments. In addition, we also tested for glutamate uptake for two reasons. First, the vesicular glutamate transporter has not yet been identified at the molecular level and no antibodies are available. Because glutamatergic vesicles represent the largest vesicle pool in mammalian brain, it was important to determine whether they were present. Second, it has been suggested that glutamate serves as the direct precursor for vesicular GABA being converted by vesicle-bound GAD in a manner that may be linked to transport. As shown in Figure 5, carbonyl cyanide $p$-trifluoromethoxyphenylhydrazone (FCCP)-sensitive uptake activity for GABA is enriched $\sim 10$-fold on the VGAT bead fraction (enrichment normalized to synaptophysin), whereas glutamate uptake activity is primarily lost.

Finally, we also monitored vesicular acidification in response to glutamate and chloride using the $\mathrm{pH}$-sensitive dye acridine orange. Previous work showed that glutamate uptake is associated with vesicular acidification, suggesting that it serves as a counterion in addition to chloride for the vacuolar ATPase (Maycox et al., 1988). As shown in Figure 6, both the starting material and synaptophysin immunoisolates showed glutamate-induced acidification. In contrast, no glutamate-induced acidification was observed in VGAT immunoisolates. Successive additions of chloride caused acidification in all fractions except the control beads, demonstrating that the loss of activity is not attributable to a loss of functionally active $\mathrm{H}^{+}$-ATPase.

\section{DISCUSSION}

In the present study, we have shown that antibodies specific for vesicular neurotransmitter transporters can be used to quantitatively immunoisolate synaptic vesicles that are specific for the transmitter, with virtually no contamination by vesicles specific for other neurotransmitters.

The vesicle subpopulation isolated with our VGAT antibodies is highly enriched for GABA uptake activity. This finding strongly supports the inference that VGAT is functioning as the GABA transporter in mammalian brain synaptic vesicles. Our data complement previous reports showing that expression of the transporter in a heterologous expression system (PC12 cells) 
leads to proton gradient-dependent GABA uptake in isolated membrane fractions (McIntire et al., 1997). Furthermore, none of the other vesicular transporters was detectable in the bead fraction, implying that the transporters cleanly define functionally distinct vesicle subpopulations that do not overlap. None of the many other synaptic vesicle proteins characterized so far show such exclusive distribution (Fykse et al., 1993; Bajjalieh et al., 1994). Particularly, it is noteworthy that incubation with radiolabeled glutamate did not result in the accumulation of radioactivity in the VGAT-specific vesicle fraction. Thus, not only do the glutamate and GABA carriers reside on separate vesicles but, at least in our preparation, there is no significant conversion of glutamate to GABA linked to transport. Although the GABAsynthesizing enzyme GAD is associated with the vesicle surface when analyzed by immunogold electron microscopy (Reetz et al., 1991), it is easily lost upon subcellular fractionation. In fact, we could not find enrichment of GAD in VGAT immunoisolates (data not shown). The apparent loss of GAD, therefore, does not allow us to assess whether GABA uptake is facilitated by coupling with its biosynthesis.

The availability of purified fractions of transmitter-specific vesicles for biochemical studies permits us to investigate differences in the transport mechanisms of individual transmitters. As outlined in the introductory remarks, vesicular uptake of glutamate, GABA, and catecholamines is dependent on different components of the electrochemical proton gradient created by the vacuolar $\mathrm{H}^{+}$-ATPase. Unlike any of the other transmitters, glutamate was shown to be cotransported with protons (Maycox et al., 1988), resulting in a transport mode in which glutamate serves as a counterion for protons in a similar manner to chloride. It has been inferred, therefore, but never proven that glutamateinduced acidification is dependent on the vesicular glutamate transporter and thus is a specific feature of glutamatergic synaptic vesicles. Our data lend strong support to this hypothesis because glutamate-dependent acidification activity was absent in purified GABAergic vesicles. In contrast, GABAergic vesicles still displayed chloride-dependent acidification, suggesting that all synaptic vesicles contain chloride channels.

Our analysis has revealed that all the major synaptic vesicle proteins for which we have tested are present on GABAergic synaptic vesicle membranes. Moreover, a comparison of general protein staining patterns on electropherograms has revealed no obvious differences in banding. This sort of uniformity of vesicle membranes was inferred previously from immunocytochemistry but has never been systematically investigated. Of course, we cannot yet exclude the possibility that GABAergic vesicles contain other idiotypic proteins that are not resolved by onedimensional electrophoresis. However, at present, our findings are consistent with the notion that synaptic vesicles are biochemically homogeneous with respect to most of their constituents and thus that the mechanisms of exocytosis and vesicle recycling are the same for all neurotransmitter vesicles. Neurotransmitter vesicles become unique by virtue of the inclusion of selective transporters in their membranes.

\section{REFERENCES}

Alfonso A, Grundahl K, Duerr JS, Han HP, Rand JB (1993) The Caenorhabditis elegans unc-17 gene: a putative vesicular acetylcholine transporter. Science 261:617-619.

Angel I, Fleissner AS (1983) Synaptic vesicles from hog brain: their isolation and the coupling between synthesis and uptake of gammaaminobutyrate by glutamate decarboxylase. Neurochem Int 5:697-712.
Bajjalieh SM, Frantz GD, Weimann JM, McConnell SK, Scheller RH (1994) Differential expression of synaptic vesicle protein 2 (SV2) isoforms. J Neurosci 14:5223-5235.

Brose N, Petrenko AG, Südhof TC, Jahn R (1992) Synaptotagmin: a $\mathrm{Ca}^{2+}$ sensor on the synaptic vesicle surface. Science 256:1021-1025.

Burger PM, Mehl E, Cameron PL, Maycox PR, Baumert M, Lottspeich F, De Camilli P, Jahn R (1989) Synaptic vesicles immunoisolated from rat cerebral cortex contain high levels of glutamate. Neuron 3:715-720.

Burger PM, Hell JW, Mehl E, Krasel C, Lottspeich F, Jahn R (1991) GABA and glycine in synaptic vesicles: storage and transport characteristics. Neuron 7:287-293.

Chapman ER, Hanson PI, An S, Jahn R (1995) $\mathrm{Ca}^{2+}$ regulates the interaction between synaptotagmin and syntaxin. J Biol Chem 270:23667-23671.

Chaudhry FA, Reimer RJ, Bellocio E, Danbolt NC, Osen KK, Edwards RH, Storm-Mathisen J (1998) The vesicular GABA transporter, VGAT, localizes to synaptic vesicles in sets of glycinergic as well as GABAergic neurons. J Neurosci 18:9733-9750.

Christensen H, Fykse EM, Fonnum F (1991) Inhibition of gammaaminobutyrate and glycine uptake into synaptic vesicles. Eur J Pharmacol 207:73-79.

De Camilli P, Harris SM, Huttner WB, Greengard P (1983) Synapsin I (Protein I), a nerve-terminal-specific phosphoprotein. II. Its specific association with synaptic vesicles demonstrated by immunocytochemistry in agarose-embedded synaptosomes. J Cell Biol 96:1355-1373.

Edelmann L, Hanson PI, Chapman ER, Jahn R (1995) Synaptophysinbinding to synaptobrevin: a potential mechanism for controlling the exocytotic fusion machine. EMBO J 14:224-231.

Erickson JD, Eiden LE, Hoffman B (1992) Expression cloning of a reserpine-sensitive vesicular monoamine transporter. Proc Natl Acad Sci USA 89:10993-10997.

Fykse EM, Takei K, Walch-Solimena C, Geppert M, Jahn R, De Camilli P, Südhof TC (1993) Relative properties and localizations of synaptic vesicle protein isoforms: the case of the synaptophysins. J Neurosci 13:4997-5007.

Gray EG (1959) Axosomatic and axodendritic synapses of the cerebral cortex: an electron microscope study. J Anat 93:420-433.

Hell JW, Jahn R (1994) Preparation of synaptic vesicles from mammalian brain. In: Cell biology: a laboratory handbook, Ed 1 (Celis JE, ed), pp 567-574. New York: Academic.

Hell JW, Maycox PR, Stadler H, Jahn R (1988) Uptake of GABA by rat brain synaptic vesicles isolated by a new procedure. EMBO J 7:3023-3029.

Hell JW, Maycox PR, Jahn R (1990) Energy dependence and functional reconstitution of the GABA carrier from synaptic vesicles. J Biol Chem 265:2111-2117.

Holtje M, von Jagow B, Pahner I, Lautenschlager M, Hortnagl H, Nurnberg B, Jahn R, Ahnert-Hilger G (2000) The neuronal monoamine transporter VMAT2 is regulated by the trimeric GTPase Go(2). J Neurosci 20:2131-2141.

Huttner WB, Schiebler W, Greengard P, De Camilli P (1983) Synapsin I (Protein I), a nerve terminal-specific phosphoprotein. III. Its association with synaptic vesicles studied in a highly purified synaptic vesicle preparation. J Cell Biol 96:1374-1388.

Jahn R, Schiebler W, Greengard P (1984) A quantitative dotimmunobinding assay for proteins using nitrocellulose membrane filters. Proc Natl Acad Sci USA 81:1684-1687.

Jahn R, Schiebler W, Ouimet C, Greengard P (1985) A 38,000 dalton membrane protein (p38) present in synaptic vesicles. Proc Natl Acad Sci USA 82:4137-4141.

Laemmli UK (1970) Cleavage of structural proteins during the assembly of the head of bacteriophage T4. Nature 227:680-685.

Liu Y, Edwards RH (1997) The role of vesicular transport proteins in synaptic transmission and neural degeneration. Annu Rev Neurosci 20:125-156.

Liu Y, Peter D, Roghani A, Schuldiner S, Prive GG, Eisenberg D, Brecha N, Edwards RH (1992) A cDNA that suppresses MPP ${ }^{+}$toxicity encodes a vesicular amine transporter. Cell 70:539-551.

Masson J, Sagne C, Hamon M, El Mestikawy S (1999) Neurotransmitter transporters in the central nervous system. Pharmacol Rev 51:439-464.

Maycox PR, Deckwerth T, Hell JW, Jahn R (1988) Glutamate uptake by brain synaptic vesicles: energy dependence of transport and functional reconstitution in proteoliposomes. J Biol Chem 263:15423-15428.

Maycox PR, Hell JW, Jahn R (1990) Amino acid neurotransmission: Spotlight on synaptic vesicles. Trends Neurosci 13:83-87. 
McIntire SL, Reimer RJ, Schuske K, Edwards RH, Jorgensen EM (1997) Identification and characterization of the vesicular GABA transporter. Nature 389:870-876.

Mugnaini E, Dahl AL (1983) Zinc-aldehyde fication for lightmicroscopic immunocytochemistry of nervous tissues. J Histochem Cytochem 31:1435-1438.

Oertel WH, Schmechel DE, Mugnaini E, Tappaz ML, Kopin IJ (1981) Immunocytochemical localization of glutamate decarboxylase in rat cerebellum with a new antiserum. Neuroscience 6:2715-2735.

Reetz A, Solimena M, Matteoli M, Folli F, Takei K, De Camilli P (1991) GABA and pancreatic $\beta$-cells: colocalization of glutamic acid decarboxylase (GAD) and GABA with synaptic-like microvesicles suggests their role in GABA storage and secretion. EMBO J 10:1275-1284.

Renick SE, Kleven DT, Chan J, Stenius K, Milner TA, Pickel VM, Fremeau Jr RT (1999) The mammalian brain high-affinity L-proline transporter is enriched preferentially in synaptic vesicles in a subpopulation of excitatory nerve terminals in rat forebrain. J Neurosci $19: 21-33$
Sagne C, El Mestikawy S, Isambert M-F, Hamon M, Henry J-P, Giros B, Gasnier B (1997) Cloning of a functional vesicular GABA and glycine transporter by screening of genome databases. FEBS Lett 417:177-183.

Schneider WJ, Slaughter C, Goldstein JL, Anderson RGW, Capra JD, Brown MS (1983) Use of antipeptide antibodies to demonstrate external orientation of the $\mathrm{NH}_{2}$ terminus of the low density lipoprotein receptor in the plasma membrane of fibroblasts. J Cell Biol 97:1635-1640.

Stenius K, Janz R, Südhof TC, Jahn R (1995) Structure of synaptogyrin (p29) defines novel synaptic vesicle protein. J Cell Biol 131:1801-1809.

Südhof TC (1995) The synaptic vesicle cycle: a cascade of proteinprotein interactions. Nature 375:645-653.

Towbin H, Staehelin H, Gordon J (1979) Electrophoretic transfer of proteins from polyacrylamide gels to nitrocellulose sheets: procedure and some applications. Proc Natl Acad Sci USA 76:4350-4354.

Whittaker VP (1984) The structure and function of cholinergic synaptic vesicles. Biochem Soc Trans 12:561-576. 